\title{
On Theoretical Study of Magnetic Behavior of Diamond Doped with Transition Metals
}

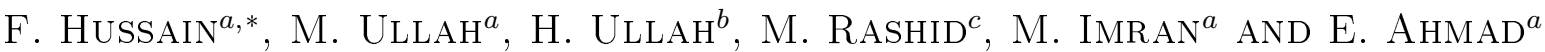 \\ ${ }^{a}$ Materials Simulation Research Laboratory (MSRL), Department of Physics, Bahauddin Zakariya University, \\ University Campus Bosan Road, 60800 Multan, Pakistan \\ ${ }^{b}$ Laser and Optronics Laboratory (LOL), Department of Physics, Bahauddin Zakariya University, \\ University Campus Bosan Road, 60800 Multan, Pakistan \\ ${ }^{c}$ Department of Physics, COMSATS Institute of Information Technology, 44000 Islamabad, Pakistan
}

(Received July 18, 2014; in final form January 15, 2015)

\begin{abstract}
We study the magnetic behavior of the diamond system. In this system diamond bulk is involved which is doped with different transition metals, namely $\mathrm{Cu}, \mathrm{Cd}, \mathrm{Hg}$, and $\mathrm{Zn}$. The VASP code is employed for all calculations which are based on density functional theory. The substitutional point defects is introduced in the diamond bulk and occupied by the transition metals. Results exhibit that all transition metals show ferromagnetism behavior and $\mathrm{Cu}$ is a good advocate of conductivity among all transition metals. The range of magnetic moments is 2.89 , 1.99, 1.96, and $1.80 \mu_{\mathrm{B}}$ per $\mathrm{Cu}, \mathrm{Cd}, \mathrm{Hg}$, and $\mathrm{Zn}$ atom in diamond bulk, respectively. Strong magnetic behavior points out that these materials could be used for spintronics.
\end{abstract}

DOI: 10.12693 /APhysPolA.127.823

PACS: 75.10.Kt, 75.10.Pq, 75.30.Hx

\section{Introduction}

For ages diamond has been an area of interest for physicists to connect its properties with various materials when doped. The interesting thing about properties of diamond is that it shows promising properties that underlies many interesting phenomenon of physics. However, it is distinguished in the sense that it exhibits optical, thermal, electronic, magnetic properties. Naturally occurring diamond does not shows magnetism but results are encouraging upon doping [1-5]. Nanocrystalline diamond films [6] improve performance of many electronic devices and their magnetic behavior can be referred to as the point to study spintronics $[7,8]$. Spintronics is a spin based concept of modifying technology which is very useful in physics. Using defects in diamond to probe magnetic properties at nanoscale has attracted many physicists in the area of the computer based simulation [911]. These experimental and theoretical research monitor ferromagnetism (FM) in carbon-based materials [11-15] and theoretical predictions about the existence of ferromagnetism in carbon-based materials induced by disorder $[16,17]$ initialized the present studies.

In this paper, we are interested in the role of substitution of transition metals (TMs) as dopant in bulk diamond crystal. In this scenario diamond lattice served to be the host and the TM takes the place in diamond lattice as the primary substituted atom. Upon doping together, their happy relationship subjects magnetism in material sciences. The TMs are assumed to be symmetrically doped in the host. When substitutional TMs oc-

*corresponding author; e-mail: fayyazhussain248@yahoo.com cupy cation sites, the $d$ states of the TMs hybridize with the $p$ states of the neighboring host anions (carbon atom). This $p-d$ hybridization results in strong magnetic interactions between localized spins and carriers in the host's valence band [18]. As a result, the system exhibits stable ferromagnetism, and the spin-polarized carriers are used for spin manipulation. We choose to study the effect of TMs metals substitution into diamond bulk. From theoretical point of view, although TMs do not have magnetic complications, and hence one can avoid possible magnetic ground states at low temperatures. In the present study we elaborate and encourage the importance of TMs doping in diamond to check out its magnetic behavior in terms of ferromagnetism.

\section{Computational methodology}

The magnetic properties of TMs doped in diamond bulk at concentrations of $1.56 \%$ are studied using super cell of $2 \times 2 \times 2$. In super cell carbon atom is substituted by TMs substitution. Here other types of defects associated with the dopants are not considered because they are strongly unfavorably as compared to the substitution. All the calculations are carried out within plane wave density functional theory by employed the Vienna $a b i n i$ tio simulation package (VASP) [19-20] which is based on density functional theory (DFT). The generalized gradient approximation (GGA) with the Perdew, Burke and Ernzerhof (PBE) of functional scheme is selected for the exchange correlation potential. The projector augmented wave (PAW) potential is selected to represent the interaction between the valance electrons and core in all these DFT calculations [21-23]. The convergence test of total energy with respect to the electron wave function is expanded using plane waves with a cut-off energy of $400 \mathrm{eV}$. The ionic position, cell volume and lattice parameters 
of the system are fully relaxed with conjugate gradient method until the Hellmann-Feynman forces are smaller than $0.02 \mathrm{eV} / \AA$ and the energy convergence criteria is met $1 \times 10^{-5} \mathrm{eV}$. The popular scheme for this calculation is Monkhorst Pack (MP) [24, 25] which is applied for $k$ point sampling. The MP grid was chosen to be $6 \times 6 \times 6$ for total energy and magnetic properties calculation for TMs doped diamond.

\section{Results and discussion}

We first optimized diamond structure and find out the optimized lattice parameter and then, we substitute one TMs atom in place of one carbon atom. This substitution causes only a slightly change in lattice parameter with $0.005 \AA$ for $\alpha$ after the structural optimization. Actually this difference occurs due to the difference in atomic radius of carbon and TMs and the change in bond length of each transition metals and four nearest neighbor carbon atoms $\left(\mathrm{TMsC}_{4}\right)$ system is around $1.54 \AA$. The spin polarized state has energies of 557,563 and 557 and $554 \mathrm{eV}$ for $\mathrm{Cu}, \mathrm{Cd}, \mathrm{Hg}$, and $\mathrm{Zn}$, respectively, lower than those of non-spin polarized state energy. The total magnetic moments are 2.89, 1.99, 1.96 and $1.80 \mu_{\mathrm{B}}$ per $\mathrm{Cu}, \mathrm{Cd}, \mathrm{Hg}$ and $\mathrm{Zn}$ atom in diamond system, respectively. The magnetization is well consistent to the Wu et al. [26] except slightly larger magnetic moments. The spin charge density of these TMs- $3 d$ and C $2 p$ is shown in Fig. 1. The spin

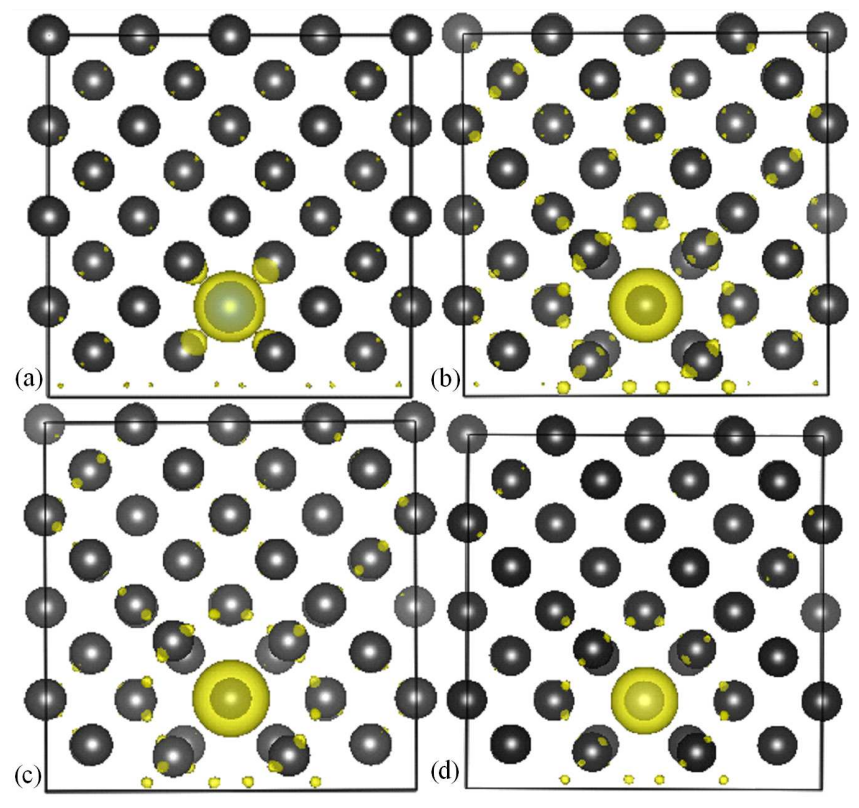

Fig. 1. Isosurface of spin charge density of diamond system doped with 1.56\%: (a) $\mathrm{Cu}$, (b) $\mathrm{Cd}$, (c) $\mathrm{Hg}$, (d) Zn.

resolved density of states of the TMs-doped diamond system is shown in Fig. 2. It can be seen from that majority spin is semiconducting with a gap and the minority spin shows metallic behavior with adequate unfilled states above the Fermi level, suggesting a half metallic behavior which is also reported in [26]. These unfilled states are very useful in charging a conduction carrier,

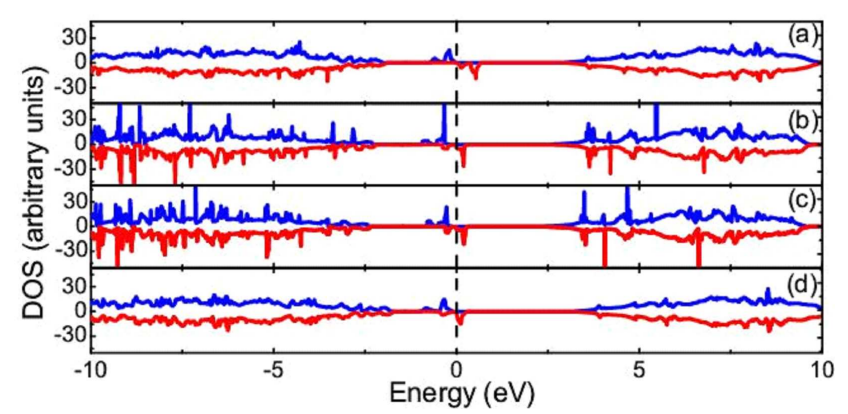

Fig. 2. Spin polarized DOSs of (a) Cu, (b) Cd, (c) $\mathrm{Hg}$, (d) $\mathrm{Zn}$ show semiconducting behavior at Fermi level.

which is required in spin injection where sufficient polarized spin current is required [27, 28], so it is proposed that TMs doped diamond can be used as spin injection charge carriers. In the present study we used the same techniques for magnetic coupling as used in [26]. For ferromagnetic and antiferromagnetic (AFM) coupling, we substitute two TMs atoms separated with largest possible distance $7.92 \AA$. The total energy is calculated for both cases along $c$-direction and it is found that FM state is the ground state.

\subsection{Electronics properties}

The spin resolved band structures of the doped diamond are shown in Fig. 3.It can be seen from the major-

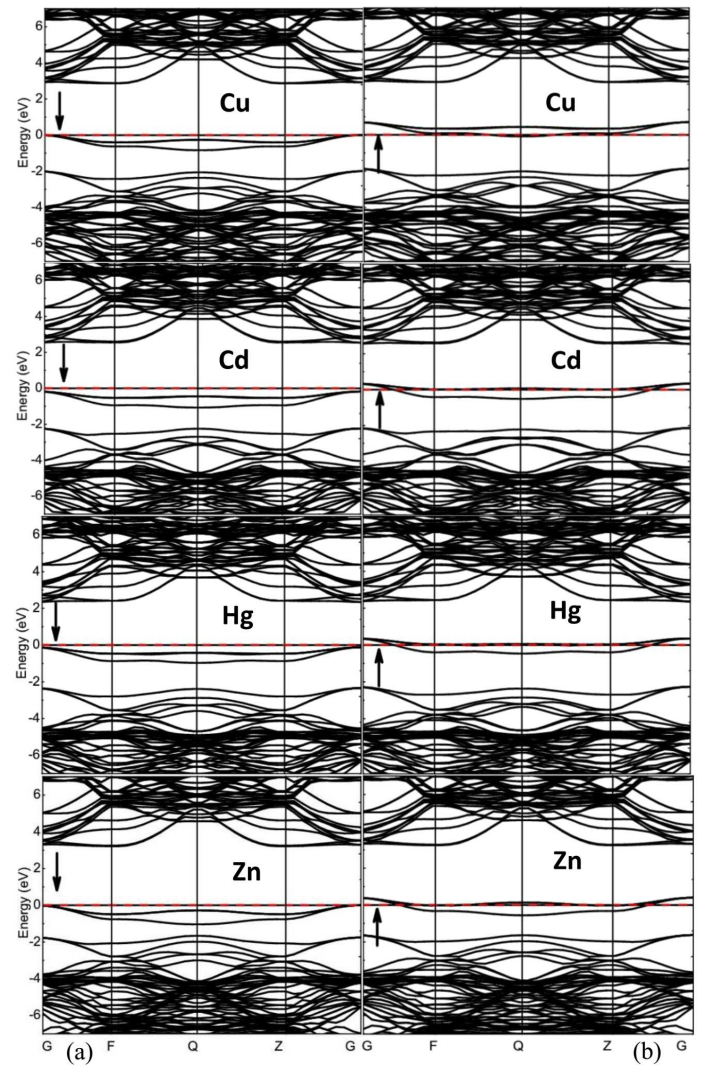

Fig. 3. Band structure of (a) minority spin, (b) majority spin of diamond doped with $1.56 \%$ of TMs and red dotted line shows the Fermi level. 
ity spin channel being semiconductor and red line shows the Fermi level in both majority and minority spin polarized band structures. The minority spin polarized band structure shows metallic behavior with adequate unfilled states above the Fermi level which is also reported in [26].It is also observed that majority spin polarized channel of $\mathrm{Cu}$-doped diamond show more semiconducting behavior and thus larger spin polarization as compared to other TMs, so the $\mathrm{Cu}$ is more favorable for this purpose.

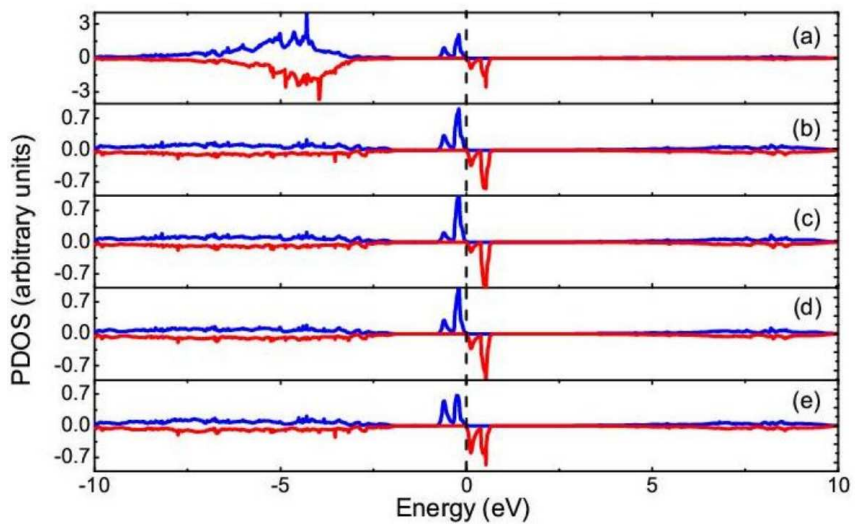

Fig. 4. Spin PDOSs of $\mathrm{Cu} 3 d$ (a) and $\mathrm{C} 2 p$ of the $\mathrm{C}$ atoms in $\mathrm{Cu}-\mathrm{C}_{4}$ structure (b)-(e).

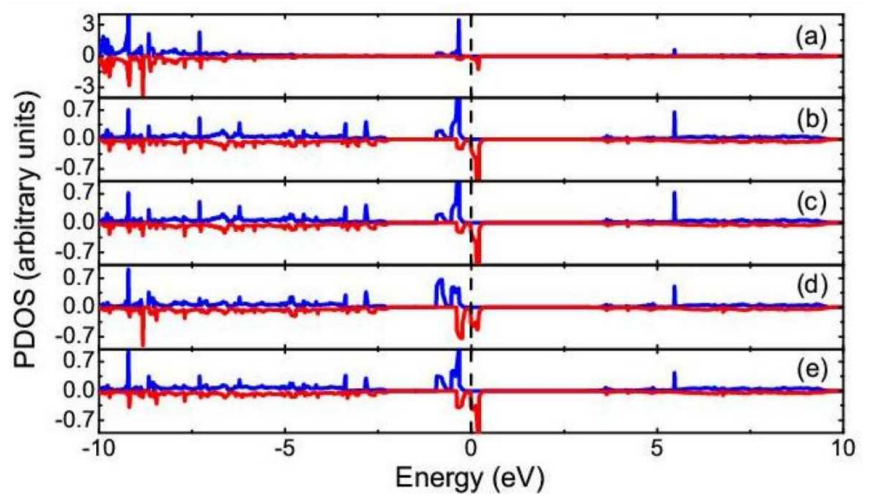

Fig. 5. Spin PDOSs of Cd $3 \mathrm{~d}$ (a) and C $2 p$ of the $\mathrm{C}$ atoms in $\mathrm{Cd}-\mathrm{C}_{4}$ structure (b)-(e).

In density of state blue and red curves correspond to $d$ and $p$ orbitals respectively for TMs doped diamond as in Fig. 2, it shows the half metallic behavior. It is observed from the results of DOS of each species having different values of curve from valence to conduction band at the Fermi level that $\mathrm{Cu}$ has higher value and this band also splits near the Fermi level $(-18.86)$ whereas $\mathrm{Zn}$ carries lowest value $(-13.46)$. The $\mathrm{Cd}$ and $\mathrm{Hg}$ consist of intermediate values. In order to understand the mechanism of stabilized FM state in TMs doped diamond, projected spin densities of states (PDOSs) of the TMs atom and its four mutual nearest neighboring $\mathrm{C}$ atoms are investigated which are shown in Fig. $4\left(\mathrm{Cu}-\mathrm{C}_{4}\right)$. The strong coupling between $\mathrm{Cu} 3 d$ and $\mathrm{C} 2 p$ can be seen from majority spin channel; the interaction peak is observed at $-2.56 \mathrm{eV}$ for

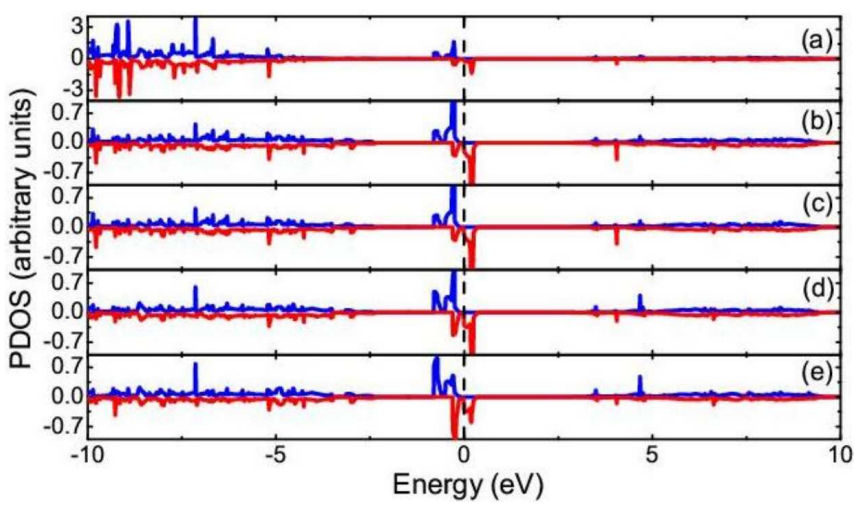

Fig. 6. Spin PDOSs of $\mathrm{Hg} 3 d$ (a) and $\mathrm{C} 2 p$ of the $\mathrm{C}$ atoms in $\mathrm{Hg}-\mathrm{C}_{4}$ structure (b)-(e).

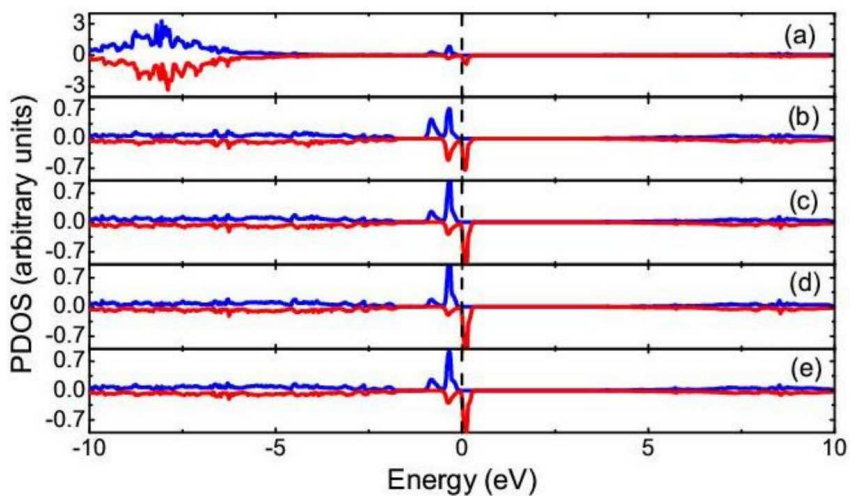

Fig. 7. Spin PDOSs of $\mathrm{Zn} 3 d$ (a) and C $2 p$ of the $\mathrm{C}$ atoms in $\mathrm{Zn}-\mathrm{C}_{4}$ structure (b)-(e).

$\mathrm{Cu} 3 d$ overlapping with that of $2 p$ of the $\mathrm{C}$ atoms in $\mathrm{Cu}-\mathrm{C}_{4}$, and the second peak of $\mathrm{Cu} 3 d$ at $-0.86 \mathrm{eV}$ also overlaps with that of $2 p$ of $\mathrm{C}$ atom with smaller magnetization. It is also observed from the valence band channel that there is splitting of states occurring in case of $\mathrm{Cu}$ atom due to $d-d$ hybridizations. In the minority spin channel the $2 p$ state of the four connecting $\mathrm{C}$ atoms con-

\section{TABLE}

Magnetic moments of different doped system in diamond.

\begin{tabular}{c|c}
\hline \hline Species & Magnetic moment $\left[\mu_{\mathrm{B}}\right]$ \\
\hline \multicolumn{2}{c}{$\mathrm{Cu}-\mathrm{C}$} \\
\hline $\mathrm{Cu}$ & 0.796 \\
$\mathrm{C}_{4}$ & 0.242 (each carbon) \\
\hline \multicolumn{2}{c}{$\mathrm{Cd}-\mathrm{C}$} \\
\hline $\mathrm{Cd}$ & 0.303 \\
$\mathrm{C}_{4}$ & 0.223 (each carbon) \\
\hline \multicolumn{2}{c}{$\mathrm{Hg}-\mathrm{C}$} \\
\hline $\mathrm{Hg}$ & 0.395 \\
$\mathrm{C}_{4}$ & 0.213 (each carbon) \\
\hline \multicolumn{2}{c}{$\mathrm{Zn}-\mathrm{C}$} \\
\hline $\mathrm{Zn}$ & 0.371 \\
$\mathrm{C}_{4}$ & 0.197 (each carbon)
\end{tabular}


tributes significantly to the unoccupied states and this kind of similar trend also be found for $\mathrm{Cd}, \mathrm{Hg}$, and $\mathrm{Zn}$ which are shown in Figs. 5, 6 and 7, respectively. These attribute indicates a strong hybridization between TMs and its four neighboring $\mathrm{C}$ atoms. This strong hybridization induces finite magnetization on TMs atom as well as the neighboring $\mathrm{C}$ atoms, as shown in Fig. 1. The each $\mathrm{C}$ atom carrying magnetization and the magnitude of the magnetization in due to all these TMs dopant in diamond is present in Table.

\section{Conclusions}

The electronic properties of TMs doped diamond have been studied by using DFT calculations. The TMs dopants become spin polarized when substitute into diamond, further this spin polarization calculation magnetizes $p$ electrons of the four neighboring carbon atoms through $p-d$ hybridization. This $p-d$ hybridization is very strong in case of $\mathrm{Cu}$ as compared to the other TMs dopants due to this $\mathrm{Cu}$-doped diamond has higher magnetic moments. This $p-d$ hybridization prefers FM coupling state among all dopants rather than that of AFM coupling. So that TMs is promising magnetic dopant for diamond to fabricate magnetic materials with reduced size and it should be free from magnetic precipitates.

\section{Acknowledgments}

This work is carried out in the Department of Physics, BZ University Multan, Pakistan with special computational facilities of Physics Department of National University of Singapore.

\section{References}

[1] E.B. Lombardi, Diam. Relat. Mater. 17, 1345 (2008).

[2] L.A. Li, S.H. Cheng, H.D. Li, Q. Yu, J.W. Liu, X.Y. Lv, Nano-Micro Lett. 2, 154 (2010).

[3] H.-X. Gao, J.-B. Li, J.-B. Xia, Physica B Condens. Matter 407, 2347 (2010).

[4] R. Kalish, Carbon 37, 781 (1999).

[5] J.P. Perdew, W. Yue, Phys. Rev. B 33, 8800 (1986).

[6] D.M. Gruen, Ann. Rev. Mater. Sci. 29, 211 (1999).
[7] H. Ohno, Science 281, 951 (1998).

[8] S.A. Wolf, D.D. Awschalom, R.A. Buhrman, J.M. Daughton, S. von Molnár, M.L. Roukes, A.Y. Chtchelkanova, D.M. Treger, Science 294, 1488 (2001).

[9] L.A. Chernozatonskii, P.B. Sorokin, A.G. Kvashnin, D.G. Kvashnin, JETP Lett. 90, 134 (2009).

[10] R. Ruoff, Nature Nanotechnol. 3, 10 (2008).

[11] R. Höhne, P. Esquinazi, V. Heera, H. Weishart, Diam. Relat. Mater. 16, 1589 (2007).

[12] K. Murata, H. Ushijima, H. Ueda, K. Kawaguchi, J. Chem. Soc. Chem. Commun. 1991, 1265 (1991).

[13] P. Turek, K. Nozawa, D. Shiomi, K. Awaga, T. Inabe, Y. Maruyama, M. Kinoshita, Chem. Phys. Lett. 180, 327 (1991).

[14] P.-M. Allemand, K.C. Khemani, A. Koch, F. Wudl, K. Holczer, S. Donovan, G. Grüner, J.D. Thompson, Science 253, 301 (1991).

[15] P. Esquinazi, A. Setzer, R. Höhne, C. Semmelhack, Y. Kopelevich, D. Spemann, T. Butz, B. Kohlstrunk, M. Lösche, Phys. Rev. B 66, 024429 (2002).

[16] P. Esquinazi, R. Höhne, J. Magn. Magn. Mater. 290-291 (I), 20 (2005).

[17] A.A. Ovchinnikov, I.L. Shamovsky, J. Mol. Struct. Theochem. 251, 133 (1991).

[18] J. González, F. Guinea, M.A.H. Vozmediano, Phys. Rev. B 63, 134421 (2001).

[19] G. Kresse, J. Furthmüller, Phys. Rev. B 54, 11169 (1996).

[20] G. Kresse, J. Furthmüller, Comp. Mater. Sci. 6, 15 (1996).

[21] J.P. Perdew, K. Burke, M. Ernzerhof, Phys. Rev. Lett. 77, 3865 (1996).

[22] C.G. Van de Walle, P.E. Blöchl, Physical Review B 47, 4244 (1993).

[23] G. Kresse, D. Joubert, Phys. Rev. B 59, 1758 (1999).

[24] H.J. Monkhorst, J.D. Pack, Phys. Rev. B 13, 5188 (1976).

[25] J.D. Pack, H.J. Monkhorst, Phys. Rev. B 16, 1748 (1977).

[26] R.Q. Wu, G.W. Peng, L. Liu, Y.P. Feng, Z.G. Huang, Q.Y. Wu, Appl. Phys. Lett. 89, 142501 (2006). 